\title{
Convergence substitution for paralysed horizontal
} gaze

\author{
Bijan Beigi, Michael O’Keeffe, Patricia Logan, Peter Eustace
}

\begin{abstract}
Three patients with paralysed horizontal gaze are presented. Involuntary use of convergence to assist horizontal gaze was noted as a late feature. All patients showed (1) unilateral or bilateral horizontal gaze palsy (two patients had one and a half syndrome, the other had bilateral nuclear sixth nerve palsies), (2) adduction of both eyes on attempted gaze into the paralysed field, (3) miosis which coincided with adduction. Convergence substitution should be considered in the differential diagnosis of gaze induced strabismus.

(Br f Ophthalmol 1995; 79: 229-232)
\end{abstract}

Positive or negative vergence is a component of most conjugate eye movements, depending on the position of the object of regard. ${ }^{1}$ Preservation of convergence in gaze palsy is well described. ${ }^{23}$ In this paper three patients with horizontal gaze paralysis are presented. All are able to use convergence to obtain a useful range of horizontal gaze into the paralysed fields. The presence of miosis in all subjects confirmed the use of convergence.

Department of Neuro-

Ophthalmology,

Beaumont Hospital,

Dublin

B Beigi

P Logan

P Eustace

Department of

Paediatric

Ophthalmology,

Children's Hospital,

Temple Street, Dublin

M O'Keeffe

Department of

Ophthalmology,

University College

Dublin at the Mater

Misericordiae

Hospital, Dublin

B Beigi

M O'Keeffe

P Logan

P Eustace

Correspondence to: Professor Peter Eustace, Department of

Ophthalmology, University College Dublin, Mater Misericordiae Hospital, Dublin 7, Ireland.

Accepted for publication 4 October 1994

\section{Case reports}

\section{CASE 1}

A 15-year-old girl presented in June 1989 with ataxia, neck stiffness, and blurring of the vision in the left eye. She had a history of glandular fever and left convergent strabismus with amblyopia. At her first ocular examination visual acuity was $6 / 9$ in the right eye and $6 / 24$ in the left eye. She had a small left convergent strabismus with left gaze palsy. Papilloedema and macular stars were noted. She also had truncal ataxia. Investigation revealed a benign cerebellar tumour with chronic hydrocephalus. The tumour was excised 1 week later. Histology reported a low grade astrocytoma. Postoperatively her vision deteriorated to $6 / 36$ in the right eye and counting fingers in the left eye. She also showed the combination of a left horizontal gaze palsy and an internuclear ophthalmoplegia (INO) looking to the opposite side (one and a half syndrome) with atrophy of the left optic disc. Four months later she started to converge on attempted left gaze (Fig 1A and B), at the same time retraction of the left globe was noted. Pupil constriction occurred synchronously with attempted gaze to the left. Infrared oculographic tracings of horizontal eye movements are shown (Fig 1C). These demonstrate the use of convergence on attempted lateral gaze to the left. Details of the findings on testing of other horizontal gaze subsystems are included in Table 1.

\section{CASE 2}

A 54-year-old woman presented in 1960 with cerebellar signs. A posterior fossa tumour was diagnosed for which she underwent craniotomies in 1960 and 1962. Postoperatively she developed right convergent strabismus with horizontal diplopia. In February 1992, she had a visual acuity of $6 / 12$ in the right eye and $6 / 5$ in the left eye. She had a right horizontal gaze palsy with an INO looking to the opposite side (one and a half syndrome). She also had a right lower motor neuron seventh nerve palsy and a right eighth cranial nerve palsy. Adduction was assisted by an active convergence accommodation effort on attempted dextroversion as shown by pupil constriction (Fig 2).

\section{CASE 3}

A 7-year-old boy who was born as a floppy baby with feeding difficulty and had an expressionless face as a result of bilateral seventh cranial nerve palsies. Bilateral nuclear sixth nerve palsies were also noted (asymmetrical horizontal gaze palsies). Moebius syndrome was diagnosed. He had a right convergent strabismus, for which bimedial recti recessions were performed at the age of 3. At the age of 5 , eye movements showed adduction with pupil constriction on attempted lateral gaze. These movements mimicked cross fixation but pupil constriction confirmed the use of vergence to assist paralysed horizontal gaze (Fig 3).

\section{Discussion}

In all conjugate eye movements, unless the object of regard is at infinity, some element of vergence is used. The information concerning both version and vergence must be synchronised. The mechanism by which this takes place is uncertain. ${ }^{1}$ Vergence, accommodation, and pupillary constriction are the components of the near triad. ${ }^{4}$ The stimuli for vergence eye movements are disparity of 


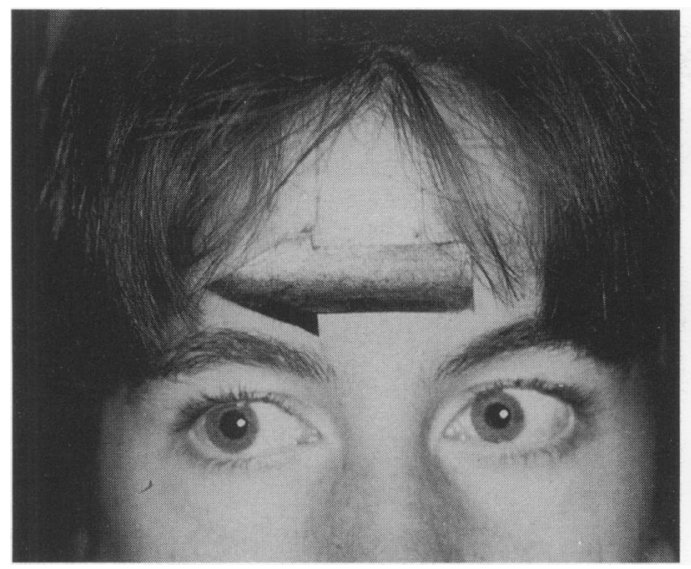

Fig $1 A$

images (fusional vergence) and retinal blur (accommodative vergence). ${ }^{1}$ ButtnerEnnever and Akert have shown the anatomical substrate of convergence in vergence eye movement. ${ }^{5}$ The area of the brain concerned with the control of convergence has been sited in the upper brainstem. ${ }^{67}$ This is influenced by cells in the visual cortex which are stimulated by binocular retinal input. Axons in the upper brainstem

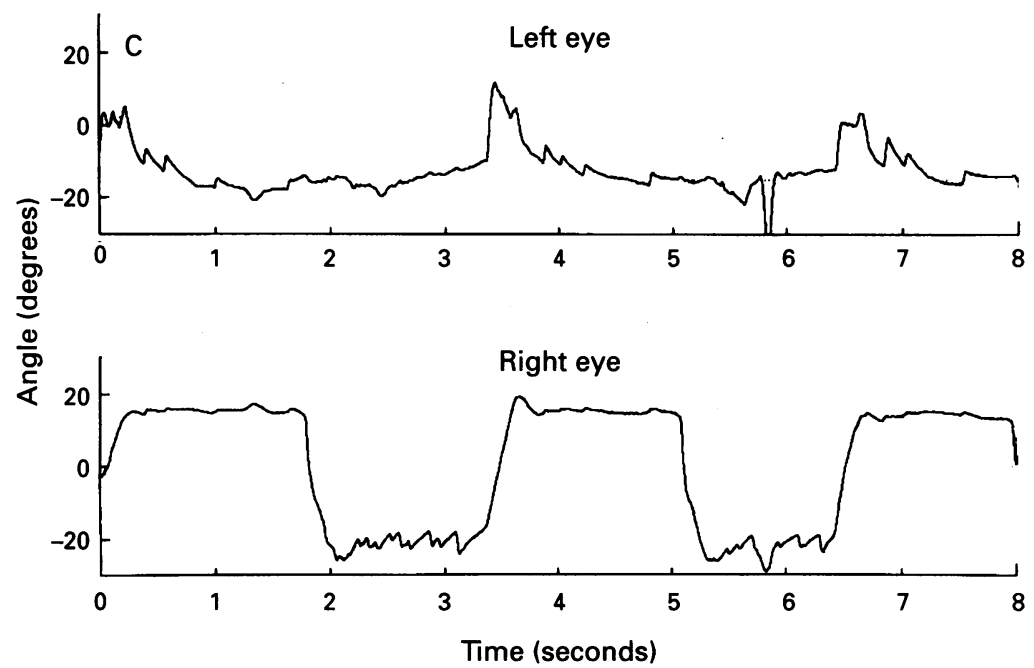

Figure 1 Horizontal ocular movements in case 1. (A) Limitation of adduction and abduction with abducting nystagmus on dextroversion. (B) Convergence and bilateral pupillary constriction in laevoversion. Convergence assists the right eye to adduct beyond the midline. (C) Infrared oculographic tracings of the horizontal eye movement in case.1.Horizontal pursuits are plotted on the vertical axis. 0 is the primary position of gaze; negative values are to the right and positive values are to the left. On attempted right gaze, at 2 seconds, the right eye shows limited abduction with gaze evoked nystagmus and the left eye shows limited adduction (internuclear ophthalmoplegia). On attempted left gaze, at 3.3 seconds, there is limited adduction of the right eye and marked limitation of abduction of the left eye. The left eye does not move beyond the primary position of gaze. Further attempts at laevoversion result in converging movements of both eyes (3.3-5 seconds). This pattern is repeated from 7 to 8 seconds.

Table 1 Ocular motility finding

\begin{tabular}{|c|c|c|c|c|c|c|}
\hline Patient & $V A$ & $\begin{array}{l}\text { Cover } \\
\text { test }\end{array}$ & $\begin{array}{l}\text { Convergence } \\
\text { used in }\end{array}$ & Horizontal pursuits & Horizontal saccades & $\begin{array}{l}\text { Vestibulo- } \\
\text { ocular reflex }\end{array}$ \\
\hline Case 1 & $\begin{array}{l}\text { R } 6 / 36 \\
\text { L CF }\end{array}$ & LCS & Left gaze & $\begin{array}{l}\text { Saccadic pursuits, } \mathrm{L} \\
\text { gaze palsy, R INO }\end{array}$ & $\begin{array}{l}\text { Hypometric, } \\
\text { reduced velocity }\end{array}$ & Defective \\
\hline Case 2 & $\begin{array}{l}\text { R } 6 / 12 \\
\text { L } 6 / 5\end{array}$ & RCS & Right gaze & $\begin{array}{r}\text { Saccadic pursuits, } R \\
\text { gaze palsy, L INO }\end{array}$ & $\begin{array}{l}\text { Hypometric, } \\
\text { reduced velocity }\end{array}$ & Defective \\
\hline Case 3 & $\begin{array}{l}\mathrm{R} 6 / 9 \\
\mathrm{~L} 6 / 9\end{array}$ & LCS & $\begin{array}{l}\text { Left and } \\
\text { right gaze }\end{array}$ & $\begin{array}{l}\text { Asymmetrical limited } \\
\text { abd + add (both } \\
\text { eyes) }\end{array}$ & $\begin{array}{l}\text { Hypometric, } \\
\text { reduced velocity }\end{array}$ & Defective \\
\hline
\end{tabular}

INO= internuclear ophthalmoplegia, LCS = left convergent strabismus, RCS = right convergent strabismus, abd=abduction, add=adduction.

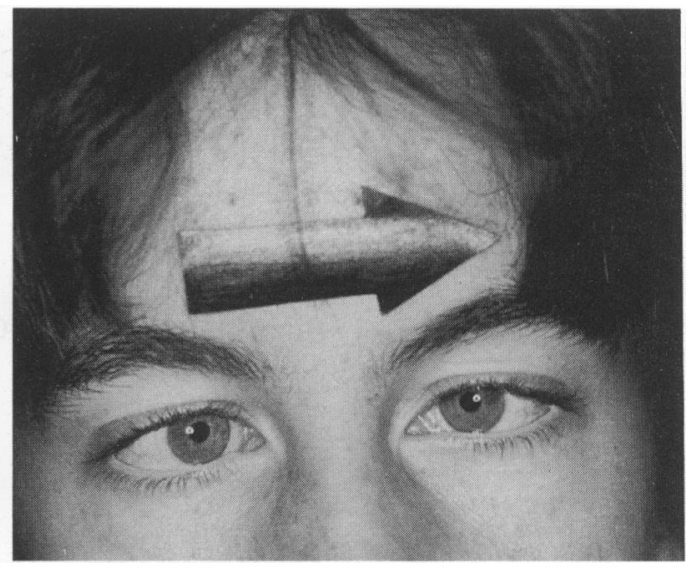

Fig $1 B$

reticular formation excite time locked cells in a motor neuron pool adjacent to the subnucleus of the oculomotor nerve for medial rectus function bilaterally. ${ }^{5}$ In a recently published model by Leigh and Zee an explanation for the addition of vergence to all eye movements, both saccadic and pursuit where the eyes are not focused on infinity, is proposed. ${ }^{1}$ Paralytic pontine exotropia in bilateral internuclear ophthalmoplegia and extropia in Webino syndrome, ${ }^{8}$ suggests co-existent involvement of vergence input to the oculomotor nuclei. ${ }^{2}$

The three cases presented here are interesting as all show the involuntary use of the vergence accommodation synkinesis when the horizontal gaze mechanism is paralysed. These cases therefore support the suggestions that both vergence eye movements driven from the upper brainstem and horizontal gaze movements driven by the pons act synergistically in all conjugate eye movements. Defect of one system can leave the other one intact (Fig 4).

In case 1 a unilateral blindness and in the other two cases suppression prevented diplopia being troublesome to the patients. Variable diplopia is a symptom of gaze induced strabismus. In such circumstances various clinical possibilities should be ruled out. ${ }^{9}$ Convergence substitution for paralysed gaze should be considered as one of the causes of gaze induced strabismus. Normally the presence of miosis myopia and vergence eye movements in the presence of any disturbance of eye movement suggests a functional condition, 'near reflex spasm'. In all our cases the use of the accommodation, convergence, miosis synkinesis was involuntary with no additional features to suggest the functional use of this triad.

The other clinical situation where miosis accompanies vergence in an abnormal way is misdirection regeneration of the oculomotor nerve, the 'pseudo-Argyll-Robertson pupil'. There was no evidence that any of our patients had a third nerve palsy at any time during their illness and therefore this possible diagnosis did not arise. Neither was there any evidence of misdirection innervation of oculomotor and abducent nerve as simultaneous constriction of the pupil is absent in such conditions. ${ }^{1011}$ 
Figure 2 Horizontal eye movements of case 2 . $(A)$ Convergence and bilateral pupillary constriction on attempted dextroversion. (B) Limitation of adduction and abduction in laevoversion, with abducting nystagmus.

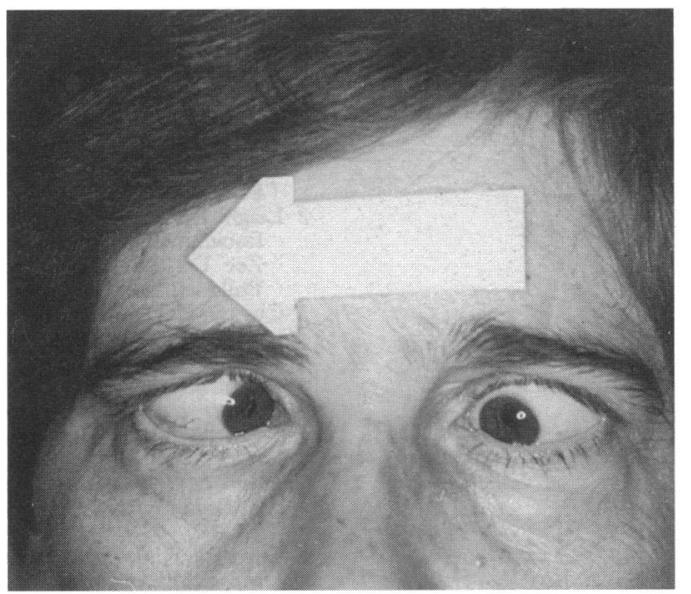

Fig $2 A$

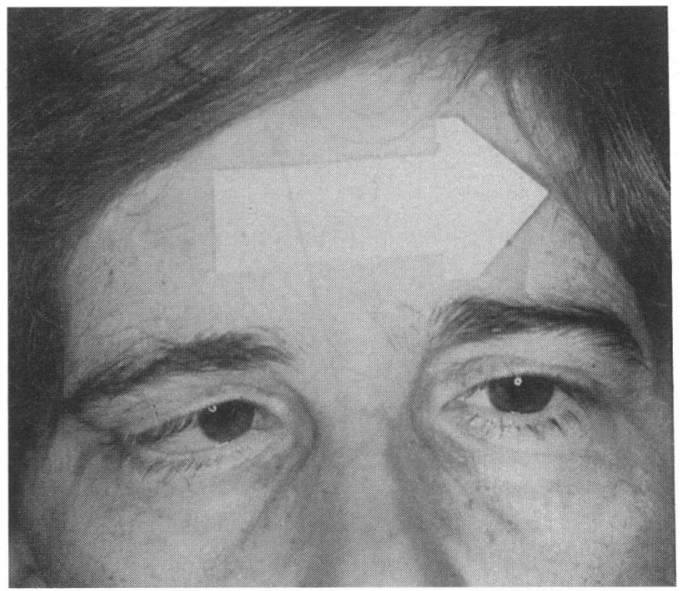

Fig $2 B$

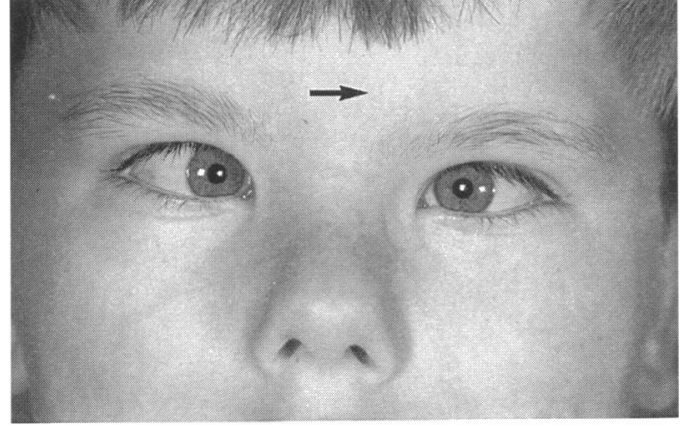

Fig $3 C$

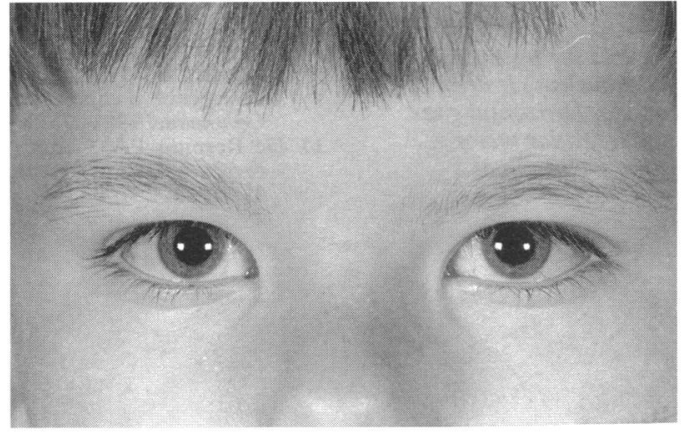

Fig $3 A$

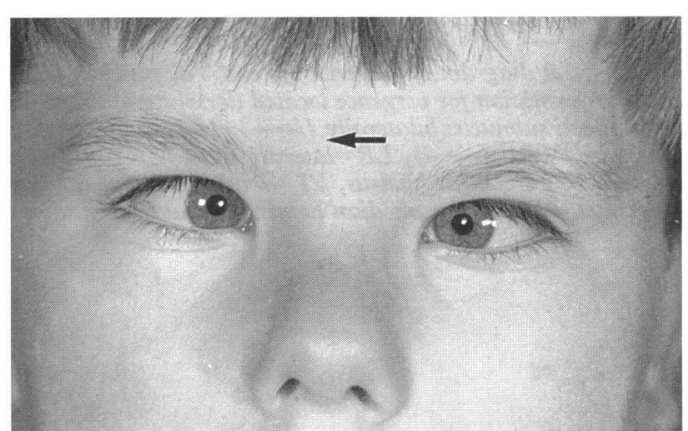

Fig $3 B$

Figure 3 Horizontal ocular movements of case 3. (A) Primary position of gaze. Note the pupil size. (B) Right gaze assisted by accommodative convergence, shown by bilateral miosis. (C) Left gaze also assisted by convergence substitution. (D) Oculographic tracing of the horizontal pursuits of case 3. At 1 second the patient has started to follow the target to the left. At 3 seconds both eyes have adducted. At 4 seconds the patient has started to look to the right. Both eyes have converged at 5 seconds.

We have reported three cases with disturbance of horizontal gaze in whom adduction with pupillary constriction in both eyes was noted on attempted gaze into the paralysed field. The vergence mechanism is used in this situation to assist horizontal gaze. This clinical entity is commoner than is recognised and should be considered in the differential diagnosis of gaze induced strabismus. This observation is also clinical support for Leigh and Zee's model of convergence suggesting synchronised co-existence of the vergence and version system in conjugate eye movements. Our patients have used the vergence system in the absence of the version system to assist limited gaze. 


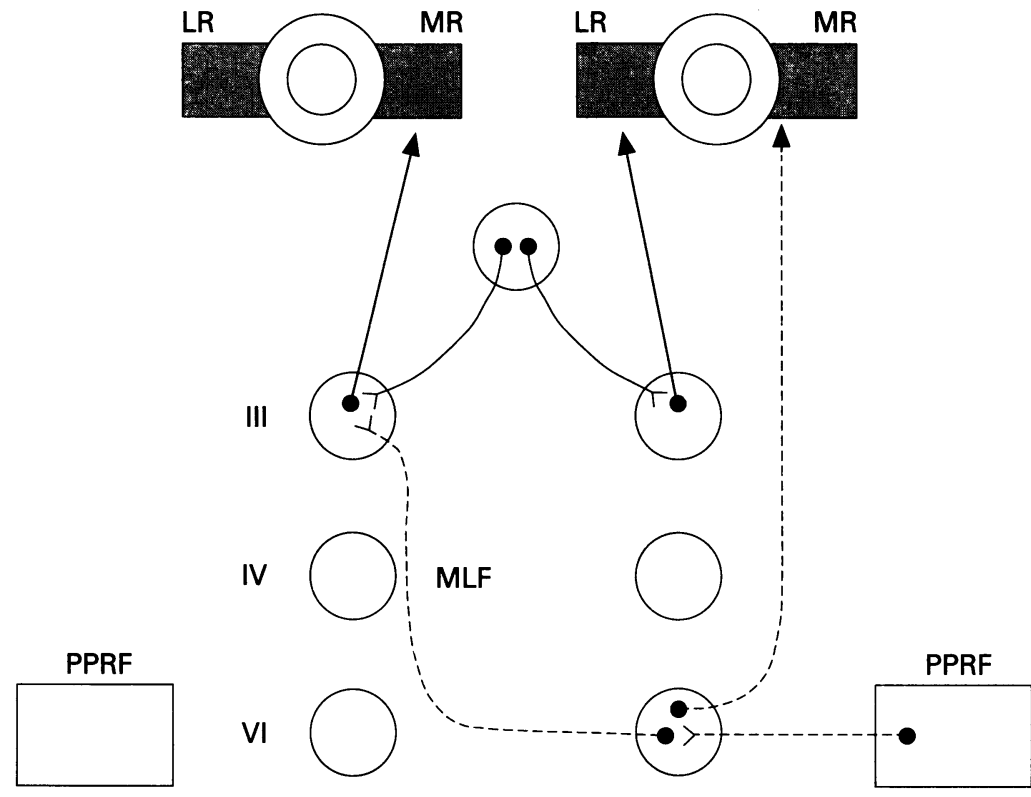

Figure 4 A diagram of vergence system. The middle nucleus represents the mesencephalic reticular formation for vergence located dorsolateral to the oculomotor nucleus. It drives medial recti subnuclei bilaterally $(-)$. Note the separate pathway for horizontal gaze driven by $P P R F(--) . L R=$ lateral rectus, $M R=$ medial rectus, III=ocular motor nucleus, $I V=$ trochlear nucleus, $V I=$ abducent nucleus, $M L F=$ medial longitudinal fasciculus, $P P R F=$ paramedian pontine reticular formation.
The authors would like to acknowledge the assistance of $\mathrm{Mr}$ Colm Saidlear for oculographic recordings.

1 Leigh RJ, Zee DS. Vergence eye movements. In: Leigh RJ, Zee DS, eds. The neurology of eye movements. 2nd ed. Philadelphia: FA Davis, 1991: 264-90.

2 Leigh RJ, Zee DS. Lesions of the medial longitudinal fasciculus: internuclear ophthalmoplegia. In: Leigh RJ, Zee DS, eds. The neurology of eye movements. 2nd ed. Philadelphia: FA Davis, 1991: 432-6.

3 Cogan DG. Internuclear ophthalmoplegia, typical and atypical. Arch Ophthalmol 1970; 84: 583-9.

4 Semmlow JL, Hung G. The near response: theories of control. In: Schor CM, Ciuffreda KJ, eds. Vergence eye control. In: Schor $\mathrm{CM}$, Ciuffreda $\mathrm{KJ}$, eds. Vergence eye
movements: basic and clinical aspects. Woburn movements: basic and clinical aspects.
Massachusetts: Butterworths, 1983: 175-95.

5 Buttner-Ennever J, Akett K. Medial rectus subgroups of the oculo-motor nucleus and their abducens internuclear input in the monkey. $f$ Comp Neurol 1981; 197: 17-27.

6 Warwick R. Representation of the extra-ocular muscles in the oculomotor nuclei of the monkey. F Comp Neurol 1953; 98: 449-503.

7 Nays LE. Neural control of vergence eye movements; convergence and divergence neurons in mid brain. $f$ vergence and divergence neu
Neurophysiol 1984; 5: 1091-108.

8 McGettrick P, Eustace P. The Webino syndrome. NeuroOphthalmol 1985; 5: 109-15.

9 Newman SA. Gaze-induced strabismus. Surv Ophthalmol 1993; 38: 303-9.

10 Cruysberg JRM, Mtanda AT, Duinkerke-Eerola KU, Huygen PLM. Congenital adduction palsy and synergistic divergence: a clinical and electro-oculographic study. $\mathrm{Br} \mathcal{F}$ Ophthalmol 1989; 73: 68-75.

11 De Respinis PA, Caputo AR, Wagner RS, Guo S. Major review - Duane's retraction syndrome. Surv Ophthalmol 1993; 38: 257-88. 